\title{
Analysis of Defects in HgCdTe and CdTe Epilayers on Si by Dual-Beam FIB
}

\author{
Taehun Lee, ${ }^{*}$ J. Huang, ${ }^{*}$ D.K. Cha, ${ }^{*}$ R.N. Jacobs, ${ }^{* *}$ J.H. Dinan, ${ }^{* *}$ and M.J. Kim* \\ * The University of Texas at Dallas, Department of Electrical Engineering, Richardson, TX 75083 \\ ** U.S. ARMY RDECOM CERDEC NVESD, Ft. Belvoir, VA 22060
}

The II-IV semiconductor alloy $\mathrm{HgCdTe}$, is the optical absorber material of choice for high performance infrared detectors. For this application, epitaxial layers of $\mathrm{HgCdTe}$ are deposited on $\mathrm{Si}$ substrates by molecular beam epitaxy (MBE). CdTe buffer layers are used to mitigate the effects of the large mismatch in lattice parameter and thermal expansion coefficient between $\mathrm{HgCdTe}$ and $\mathrm{Si}$ [1]. High defect densities have been revealed in both CdTe and $\mathrm{HgCdTe}$ epilayers grown on Si. To reach the theoretical performance of $\mathrm{HgCdTe}$ detectors, it is necessary to study the nature and origin of these defects as a first step toward eliminating them.

Conventional TEM sample preparation involving Ar+ ion milling is problematic for CdTe and related samples which are soft, fragile and highly susceptible to surface damage. Conventionally prepared TEM samples are also of limited value in locating specific defects throughout epilayers that are often $10-20 \mu \mathrm{m}$ thick. FIB with its ability to prepare site-specific TEM samples should be a more controlled and effective method. There have been no previous reports on the application of FIB to $\mathrm{HgCdTe}$. In the work reported here, an FEI Nova NanoLab 200 equipped with five gas injection sources for deposition and etching and a JEOL 2100F field-emission TEM were used. Defects (including oval hillocks and large circular clusters) are first located in-situ by SEM and ionmilled into cross-section by FIB. The morphology and the origin of defects can be analyzed by acquiring high-resolution SEM images in sequence during FIB.

Figure 1 shows an SEM image of a single hillock defect on a CdTe surface (a), which is milled into cross-section using FIB (b). The images show that the defect originates within the CdTe layer and not at the $\mathrm{CdTe} / \mathrm{Si}$ interface, a finding that is of utmost importance to an understanding of the MBE process, and one that has been observed elsewhere [2]. For a large circular cluster shown in Fig. 2(a), the regular cross-sectioning FIB recipe is of little value due to its size which is about $0.5 \mathrm{~mm}$ in diameter. In this case, the ion-beam imaging with a large beam current appears to be more practical. Fig. 2(b) illustrates that three regions shown in Fig. 2(a) exhibit distinctively different surface morphology, defects density and side wall profiles. To examine dislocations in a composite $\mathrm{HgCdTe} / \mathrm{CdTe} / \mathrm{Si}$ structure, a TEM sample was prepared by FIB to allow all interfaces of interest to be milled to electron transparency throughout the $15 \mu \mathrm{m}$ thick epitaxial structure. It has been reported earlier $[3,4]$ that CdTe based samples prepared by conventional Ar+ ion milling, show less ion-induced surface damage when followed by an iodine milling step. We investigated this possibility for FIB sample preparation and found that an iodine-enhanced milling process can greatly reduce the surface damage observed compared to that observed when FIB thinning is carried out with Ga+ ions alone. Fig. 3(a) shows an SEM image with (left half) and without (right half) the use of iodine. A TEM image of the $\mathrm{HgCdTe} / \mathrm{CdTe}$ interface is shown in Fig. 3(b), with the smoother surface on the left and rougher surface on the right. Dislocations and other growth defects both at the interface and within the epilayers are more clearly visible after iodine-enhanced etching. To our knowledge this is the first report and demonstration of an iodine-enhanced FIB preparation technique for $\mathrm{HgCdTe}$ and CdTe TEM specimens [5]. 


\section{References}

[1] O.K. Wu et al., Narrow-gap II-VI Compounds for Optoelectronic and Electromagnetic Applications, Chapman \& Hall, London, 1997.

[2] E.M. Campo et al., J. Electron. Materials, 34 (2005) 953.

[3] A.G. Cullis, N.G. Chew and J.L. Hutchinson, Ultramicroscopy 17 (1985) 203-212.

[4] N.G. Chew and A.G. Cullis, Ultramicroscopy 23 (1987) 175-198.

[5] This research is supported by U.S. ARMY RDECOM CERDEC NVESD.
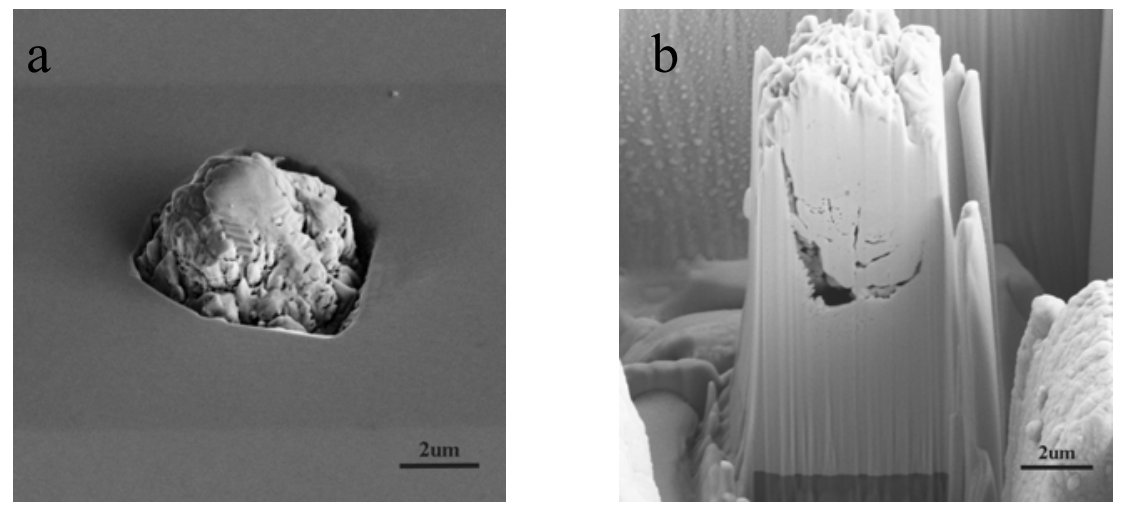

FIG. 1. (a) SEM image of an oval hillock defect on the CdTe surface. (b) The defect is revealed in three-dimension after FIB removed the surrounding material.
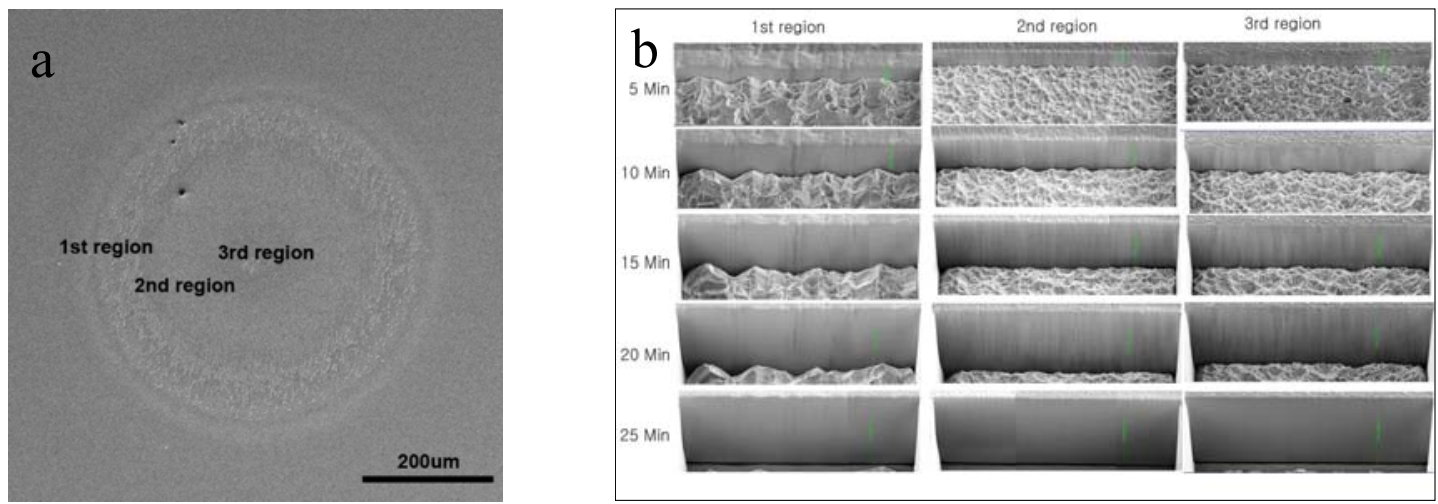

FIG. 2. (a) The circular defects are observed on the CdTe surface. (b) The SEM images of the sidewall profiles from three regions indicated in (a) with increasing ion-beam exposure time.
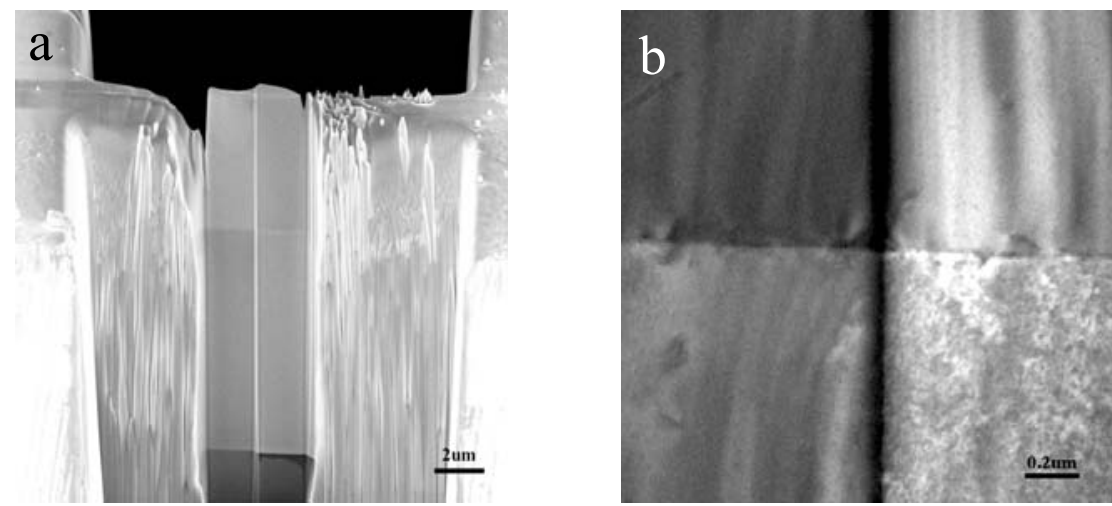

FIG. 3. (a) SEM image of $\mathrm{HgCdTe} / \mathrm{CdTe} / \mathrm{Si}$ multilayer with (left) and without (right) the use of iodine-enhanced ion-mill. (b) TEM image showing smoother and clearer surface on the left. 